SSCL-555

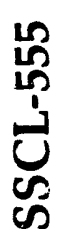
Superconducting Super Collider Laboratory

Super Slow Extraction at the SSC Using Channeling in a Curved Crystal

\author{
B. S. Newberger, H.-J. Shih, \\ and J. A. Ellison
}

October 1991 


\title{
Super Slow Extraction at the SSC Using Channeling in a Curved Crystal
}

\author{
B. S. Newberger \\ Institute for Fusion Studies \\ The University of Texas at Austin \\ Austin. Texas 78712 \\ H.-J. Shih \\ Superconducting Super Collider Laboratory* \\ 2550 Beckleymeade Avenue \\ Dallas, Texas 75237 \\ J. A. Ellison \\ Department of Mathematics and Statistics \\ The University of New Mexico \\ Albuquerque, New Mexico 87131
}

October 1991

\section{DISCLAIMER}

\begin{abstract}
This report was prepared as an account of work sponsored by an agency of the United States Government. Neither the United States Government nor any agency thereof, nor any of their employees. makes any warranty. express or implied. or assumes any legal liability or responsibility for the accuracy, completeness, or usefulness of any information, apparatus. product, or process disclosed. or represents that its use would not infringe privately owned rights. Reference herein to any specific commercial product, process, or service by trade name. trademark, manufacturer. or otherwise does not necessarily constitute or imply its endorsement, recommendation. or favoring by the United States Government or any agency thereof. The views and opinions of authors expressed herein do not necessarily state or reflect those of the United States Government or any agency thereof.
\end{abstract}

\footnotetext{
- Operated by the Tiniversitses Research Association. L.e. for the L S Defartment of Energy under ('ontract No. DE-AC3 J-89ERtJ486 
SSCL-5 55

\title{
Super Slow Extraction at the SSC Using Channeling in a Curved Crystal
}

\author{
B. S. Newberger, H.-J. Shih, and J. A. Ellison
}

\begin{abstract}
The possibility of a high-precision $B$-physics experiment in a fixed target configuration has stimulated considerable interest in the extraction of a low-intensity proton beam from the SSC during collider operation. The candidate scheme which has received the most attention uses a bent crystal of Si to deflect protons into the extraction line. In this paper. we present results on deflecting efficiency of Si (110) planes and on the feeding of the crystal by controlled injection of noise into the collider rf system. These results are important in estahlishing the viability of simultaneous collider and fixed target operation.
\end{abstract}




\subsection{INTRODUCTION}

As its name implies, the Superconducting Super Collider is a hadron storage ring whose principal objective is to explore $\mathrm{TeV}$ mass scale physics in high-p $p_{t}$ collisions of intersecting counter-rotating proton beams. However, the high energy of the stored protons, $20 \mathrm{TeV}$. the highest value of any machine now contemplated. makes possible other interesting physics. One concept which has been receiving attention is a fixed target experiment for the investigation of heavy flavors, particularly high-statistics $B$ meson physics. The Super Fixed Target (SFT) experiment ${ }^{1}$ as it has come to be called exploits the relatively: long decay length of a $B$ meson in the laboratory frame to simplify reconstruction of the candidate events. Detector data handling limits determine the proton extraction rate into the beamline. This is small, of the order of $10^{8}$ pps. which is comparable to the rate at which protons would be removed at a high luminosity $\operatorname{IR}\left(\mathcal{L}=10^{33} \mathrm{~cm}^{2} \mathrm{sec}^{-1}\right)$ and is a fraction of the rate of conventional slow spill on hadron storage rings like the Tevatron. It is conceivable then, to operate such an experiment in conjunction with collider operations. This precludes the usual slow spill techniques like resonant extraction and, at SSC energies. conventional extraction hardware would be difficult to implement in any case. For these reasons, we have been exploring the possibility of a novel approach using the phenomenon of planar channeling of charged particles in curved single crystals of $\mathrm{Si}^{2}{ }^{2}$ The original suggestion that channeling in such crystals might be applied to the optics of particles in accelerators is due to Isy.ganov. ${ }^{3}$ Subsequently, this has been demonstrated in sereral applications most of which are reviewed in Reference 2 . The most recent example has been at the SpS HS beamline. ${ }^{4}$ In this paper, we discuss our recent theoretical and computational results on the extraction of the low intensity beam. The two fundamental issues which must be confronted are the efficiency of the channeling and the repopulation of the beam phase space cut by the crystal which. of necessity, must lie in the tail of the beam distribution.

In the remaining part of the introduction. we provide a brief description of the extraction geometry as it is now enrisioned relative to the SSC footprint. We then turn to the questions of dechanneling and phase space repopulation in the subsequent two sections. A concluding section discusses the direction of future work.

The SSC lattice includes two utility straights, one in the west campus which provides space for injection. acceleration and beam dumping. and one in the east campus. At one stage of the conceptual design. it was anticipated to allow for beam dumps in both utility straights. While the site-specific design provides for beam absorbers only in the West campus the companion utility straight with its closed orbit bump remains in the Fast rampus lattice. This is a natural location for the low-intensity extraction line. Planar 
channeling in a bent crystal of $\mathrm{Si}$ is used to provide the kick into the field-free region of the Lambertson string, as illustrated in Figure 1. To deflect the extracted protons into the gap in the distance shown requires an angular kick of slightly less than $100 \mu \mathrm{rad}$.

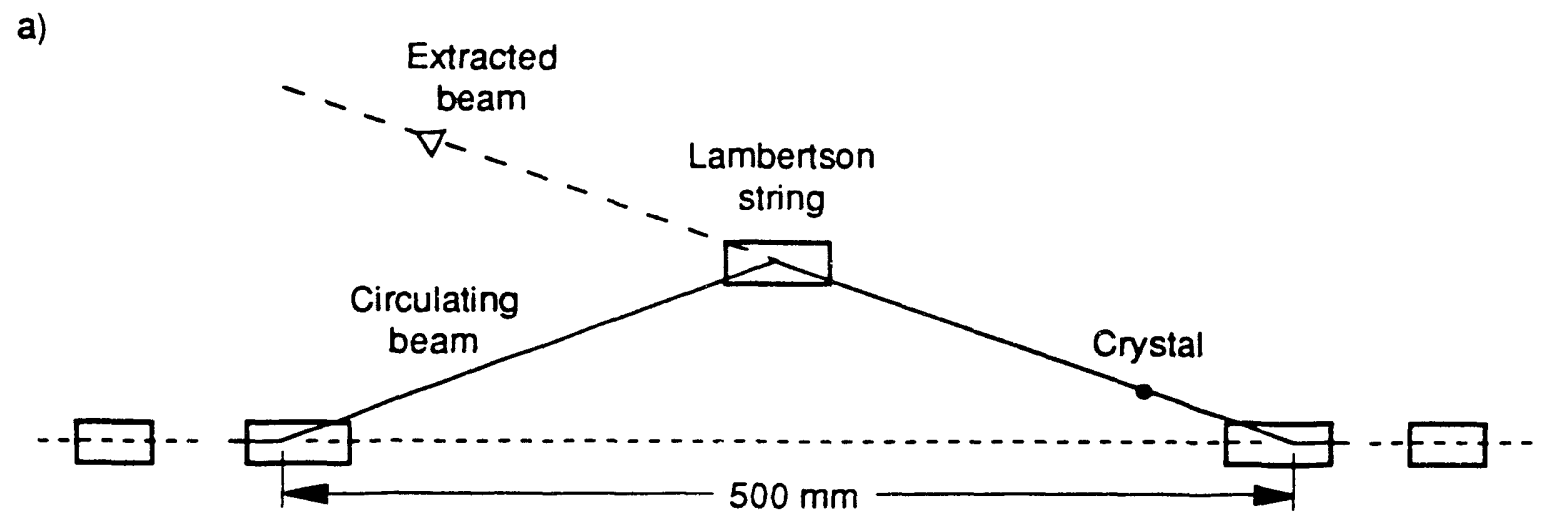

b)

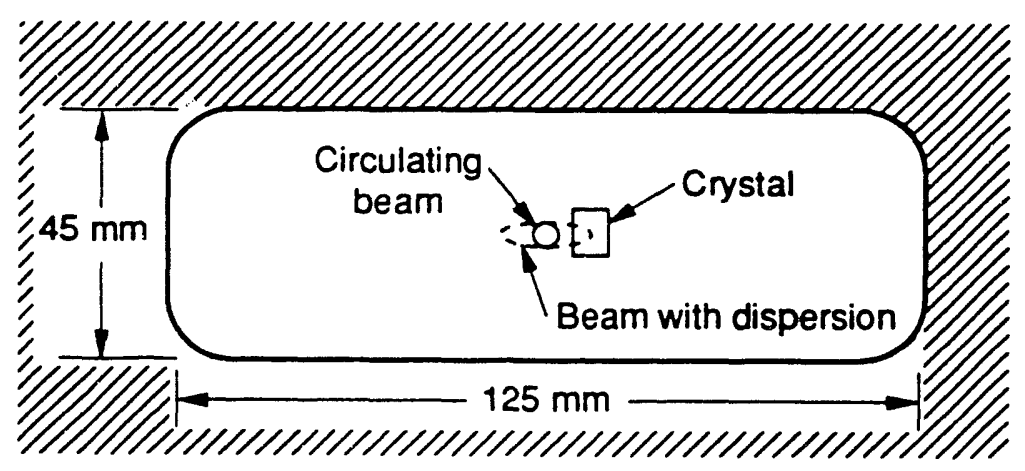

c)

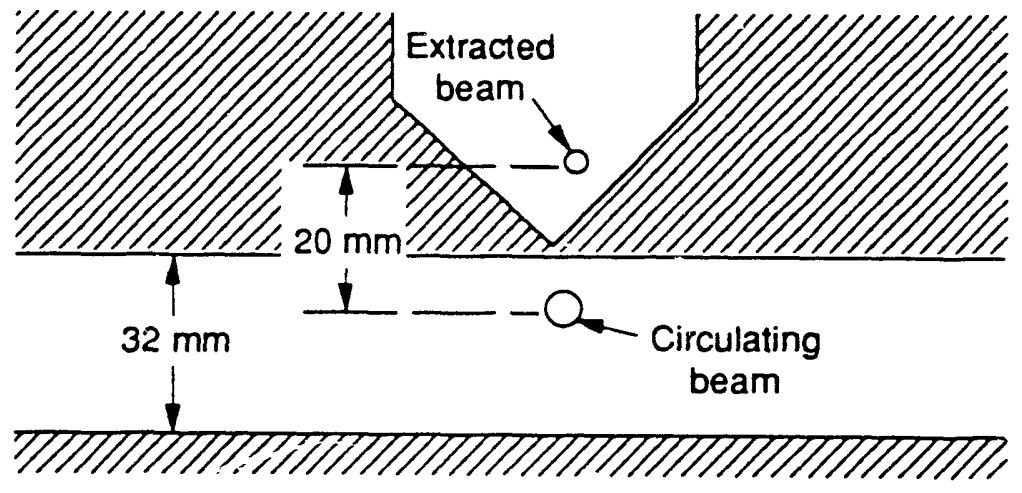

TIP.01928

Figure 1. Schematic Illustration of the Extraction Utility. (a) Extraction closed orbit bump. (t) Cross-section at the crystal. (c) Cross-section at the Lambertsons.

In our extraction geometry. the channeling crystallographic planes are horizontally oriented with the bend in the vertical direction: that is, the radius of curvature lies in a 
vertical plane tangent to the closed orbit. While tiis fits naturally into a horizontal machine. it was originally suggested, ${ }^{5}$ as a way tu alleviate a concern with the ability to orient the channeling crystallographir planes with the physical surface of the crystal. This could be a difficulty with a deflection in the horizontal piane. The quantitative requirements which are imposed on the extractor will be included in our discussion of the extraction mechanics to which we now turn.

\subsection{CRYSTAL CHANNELING}

The unique feature of the approach to extraction in the SFI is the use of channeling in a bent single crystal of Si to provide the kick which allows the extracted protons to clear a magnetic septum. There has been much work done on the channeling of relativistic particles of various "species" in both axial and planar channels and in both straight and curved crystals. (A review of the subject is provided in the collection of papers "Relativistic Channeling," cf. Reference 2). Space does not permit a detailed discussion and. in any case. most of the new results we wish to report do not involve the channeling process.

It is reasonable to use the (110) planes of $\mathrm{Si}$ to deflect the particles and the critical angle for $20 \mathrm{TeV}$ protons is $\approx 1 \mu \mathrm{rad}$. At the proposed crystal position in the SSC lattice, the beam divergence is $\approx \frac{1}{3} \mu \mathrm{rad}$ so one expects a significant fraction of the protons which hit the crystal to be channeled. To obtain an estimate of this fraction as well as the fraction of the particles which hit the crystal and are fully deflected. we use the model presented in Reference 6 and further discussed in Section 4 of Reference $i$. Particles striking the crystal are uniformly distributed in space between two adjacent crystal planes and Gaussian distributed in angle with variance $\approx(0.30 \mu \mathrm{rad}) .^{2}$ We consider the crystal to be bent with a constant curvature with a very short straight section preceding the bend. We take a radius of curvature of $300 \mathrm{~m}$ corresponding to $100 \mu \mathrm{rad}$ over $3 \mathrm{~cm}$. The fraction of particles channeled in the straight portion is then calculated by assuming a particle dechannels if it penetrates too close to a plane. This defines a boundary in the transverse phase space of the channeled particles inside of which all channeled particles lie. The boundary is an energy surface. The channeled fraction is given by the density integrated over this phase area. In the region of constant curvature. the boundary is contracted due to the centrifugal potential and the phase area of chan...led particles is reduced. The fraction of particles fully deflected is given by the density integrated over this phase area. These are illustrated in Figure $1 \mathrm{~b}$ of Reference 6 where they are referred to as $O_{0}$ and $O_{2}$ respectively. A full discussion of the theory appears there. We use $2 . j \rho_{1}$ as our dechanneling criterion as discussed in Reference $T$. Here $\rho_{1}=0.07 \mathrm{~A}$ is the one-cimensional rms lattice vibration amplitude at room temperature. There is both 
experimental and computer simulation evidence for the $2.5 \rho_{1}$ criterion. In Figure 2, we show both of these as a function of $\Gamma=\frac{1}{2 \pi z_{1} z_{2} n e^{2}}\left(\frac{p v^{\prime}}{R}\right)$ assuming beam divergence and momentum are fixed. Here $p$ is the particle momentum, $v$ its velocity $(\approx c), R$ the bend radius, $z_{1}$ and $z_{2}$ the atomic numbers of the particle and channeling medium respectively, and $n$ the areal number density ( $=N d_{p}$, with $d_{p}$ the interplanar spacing and $N$ the atomic density). $\Gamma$ is roughly the ratio of the centrifugal potential to the channel potential. For our parameters $\left(z_{1}=1, z_{2}=14, n=0.096 \AA^{-2}, \epsilon^{2}=14.4 \mathrm{eV}-\AA, \Gamma=5.48 \times 10^{-2}\right)$, we find $78 \%$ initially channeled and $64 \%$ fully deflected.

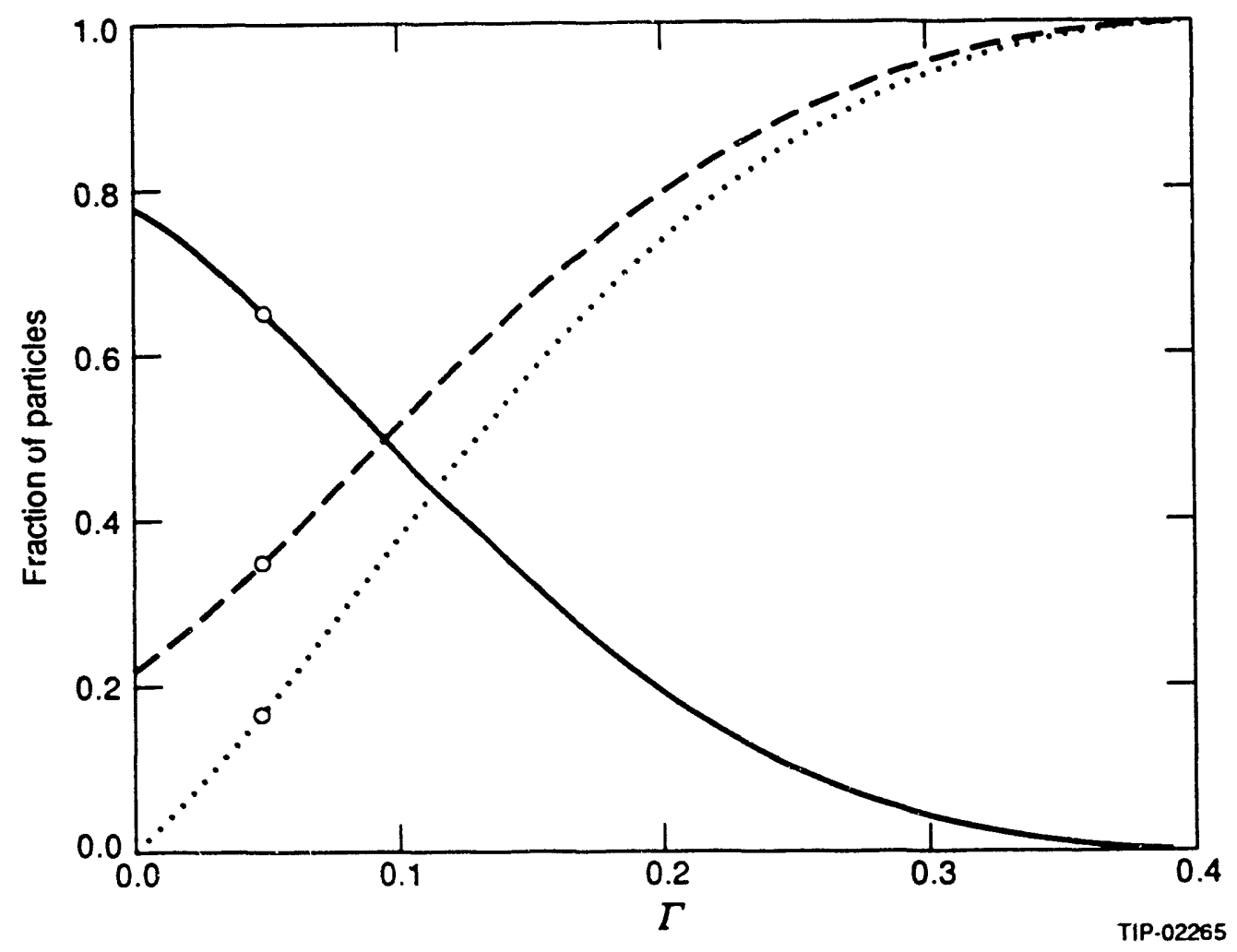

Figure 2. Planar Bending Dechanneling Curves as a Function of $\Gamma$. Dotted curve: fraction dechanneled in curved section, dashed curve: net fraction dechanneled, solid curve: net fraction channeled. The circles correspond to SSC parameters.

The next dechanneling process to consider is that due to electron multiple scattering (ems). If we assume an average electron density of $z_{2} \mathrm{~N}$ in the (110) planes of Si then the mean square scattering angle per unit length due to ems is $\approx 6.44 \times 10^{-23} \mathrm{rad}^{2} / \AA$ giving a scattering angle of $\approx 0.14 \mu \mathrm{rad}$ in $3 \mathrm{~cm}$ of $\mathrm{Si}^{8}$. Thus the ems dechanneling should be small. (For comparison. we note that the combined nuclear and electron multiple scattering in a random direction gives an rms scattering angle of $\approx 0.40 \mu \mathrm{rad}$ for $3 \mathrm{~cm}$ ). Other factors such as defects (e.g. dislocations) should be small in the Si sample sizes required. The effects of radiation damage and local heating depend on the details of the extraction 
and we will say more about this in the next section. In any case, they can be mitigated against. Thus it seems a $50 \%$ efficiency represents a not unreasonable expectation. This analysis is consistent with the CERN experiment at $450 \mathrm{GeV}^{\circ}$. Another approach to the investigation of channeling can be found in Reference 9 .

\subsection{NOISE IN THE RF SYSTEM}

The particles which are removed from the circulating beam by the crystal occupy a volume of phase space which must be renopulated if extraction is to be continuous. There is a slow growth of beam halo which occurs both from interactions at the collision points as well as from scattering on the residual gas in the beam pipe. The rate is dependent on the luminosity and does not appear to be adequate at $\mathcal{L}=10^{33} \mathrm{~cm}^{2} \mathrm{sec}^{-1}$ for the physics to be done. (For $\mathcal{L}=10^{34}$, the halo rate is likely to be sufficient but it might still be desirable to have a controlled way to move particles to the edge of phase space.) We have been investigating the use of noise injected into the rf system as a mechanism for doing this. Dispersion at the position of the crystal translates momentum displacements into transverse displacements. Two important requirements which any scheme used to bring protons onto the crystal must satisfy are considered here. The beam core in the longitudinal phase space must not be disturbed and the hit distribution must lead to the desired extraction rate. In the results to be presented. we will demonstrate that injection of suitably tailored noise can satisfy both of these.

We have investigated the effect of rf noise on the circulating SSC beam for extraction using both a diffusion theory in longitudinal action and tracking studies in the linear lattice with the noise in the rf simulated by a Monte Carlo scheme. The theoretical model is based on work at CERX and Brookhaven. ${ }^{10,11}$ Our own contributions to the development of the theory will appear soon. ${ }^{12}$ A concise description of both the theory and the simulation technique appears in Reference 13. The theory considers only the longitudinal degree of freedom while the tracking studies follow the full six-dimensional motion until a given track strikes the crystal. Nevertheless, we find good qualitative agreement between the two. We have considered both phase and amplitude noise. Phase noise typically has a larger effect on the core than does amplitude noise. For a white noise spectrum. the diffusion coefficient for the small amplitude particles is linear in th action for phase noise while quadratic for amplitude noise. However, we will see that by filtering the noise spectrum. the diffusion coefficient can be made to have a higher order tangency for these protons. with a concomitant reduction in diffusion. even for phase noise. Our results for amplitude noise will be described elsewhere. ${ }^{14}$ Tracking studies are important to interface the evolving halo with the crystal channeling. We now turn to a discussion of the important results. 
The distribution in action is shown in Figure 3a for phase noise with a notch filtered spectrum. The dashed curve gives the initial distribution in action. The solid curve and histogram respectively show the results from theory and simulation at $5 \times 10^{6}$ turns, the end of the run. The agreement between the two is good. Detailed discussion of the theoretical model and results therefrom will appear in Reference 14. Computational constraints require a reduced number of turns in the tracking, so the variance of the noise is chosen to give an adequate number of strikes on the crystal. Nevertheless, the noise is small and at the desired extraction rate at the SSC, $1-2 \times 10^{8} \mathrm{pps}$, the variance would be smaller still. It is important to verify that the core of the longitudinal distribution is preserved. A scatter plot of the longitudinal $\delta$ - $\ell$ phase space appears in Figure $3 \mathrm{~b}$, where $\delta$ is the relative momentum deviation and $\ell$ is the path length deviation. Here the initial phase coordinates of the tracks which strike the crystal are shown. The hole in the phase space in the filtered noise is a dramatic illustration of the preservation of the longitudinal core. This is also seen in Figure 3a where the change in distribution is seen to be small for $j \lesssim 0.24$. Filtering is seen to be an effective way to reduce the diffusion from the core. By contrast, white noise transports particles from the longitudinal core and would not be acceptable. ${ }^{13}$

The distribution of hits on the crystal is of considerable interest for the design of the deflecting crystal. Due to the dispersion, the noise induces a diffusion of the closed orbit. A diffusion in the horizontal position then results from a superposition of this on the betatron motion. Consider a proton which just skims the crystal when its $x$-betatron ellipse is moving 'oward the crystal. It next encounters the crystal at a subsequent turn which is tune dəpendent. Therefore the net displacement of the closed orbit, which is approximately the step size, also depends on the tune. Thus the number of hits and their distribution on the crystal are expected to depend on the size of $\beta_{x}$ and on the tune. In Figure $4 \mathrm{a}$ are shown the hits for two values of $\beta_{x}, 346 \mathrm{~m}$ and $1385 \mathrm{~m}$. The first value is representative of our earlier results ${ }^{13}$ and corresponds to the value of $\beta_{x}$ before the recent interchange of the direction of the two counterpropagating beams. The difference in $\beta_{x}$ at the location of the crystal is a consequence of the asymmetry of the utility straight with $4 \mathrm{~m}$ dispersion. We see that the distribution of hits is not especially sensitive to $\beta_{x}$ but the total number of strikes increases significantly. In both cases, the extraction rate is larger than needed in practice and the actua' noise variance would be smaller. The tune dependence is illustrated in Figure $4 \mathrm{~b}$ where the distribution is shown for a case in which the fractional part of the horizontal tune was (unrealistically) chosen close to $1 / 2$. As expected, the hit distribution became considerably narrower. Since machine operation avoids low order rationals, the effect of tune is not anticipated to be a problem. It is 

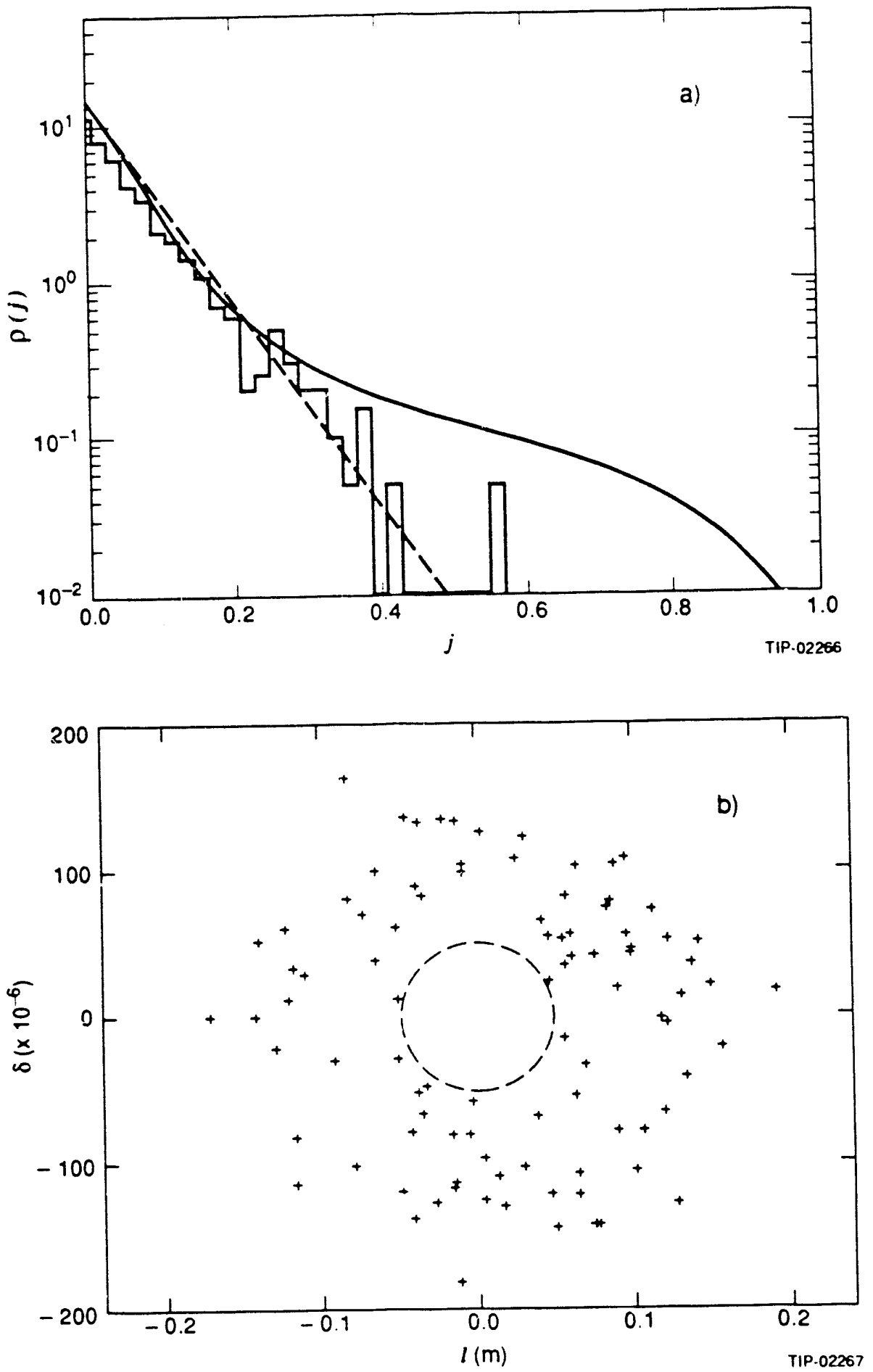

Figure 3. (a) Longitudinal Distribution in Normalized Action $j=J / J_{c}$ Where $J_{c}$ is the Action at the Image of the Crystal Position. Initial distribution is dashed. After $5 \times 10^{\epsilon}$ turns, with phase noise of variance $\sigma_{e}=0.155$ and spectrum notched in a small band around small amplitude synchrotron frequency, the solid curve gives the theory and the histogram the result of tracking simulation in sixdimensional phase space initially following 1000 particles. (b) Scatter Plot of Initial Longitudinal Phase Coordinates of 128 Tracks Which Strike Crystal. The ellipse is centered at $(0,0)$ with major and minor axes given by $\sigma_{\delta}=5.0 \times 10^{-5}$ and $\sigma_{\ell}=5.1 \times 10^{-2} \mathrm{~m}$. 

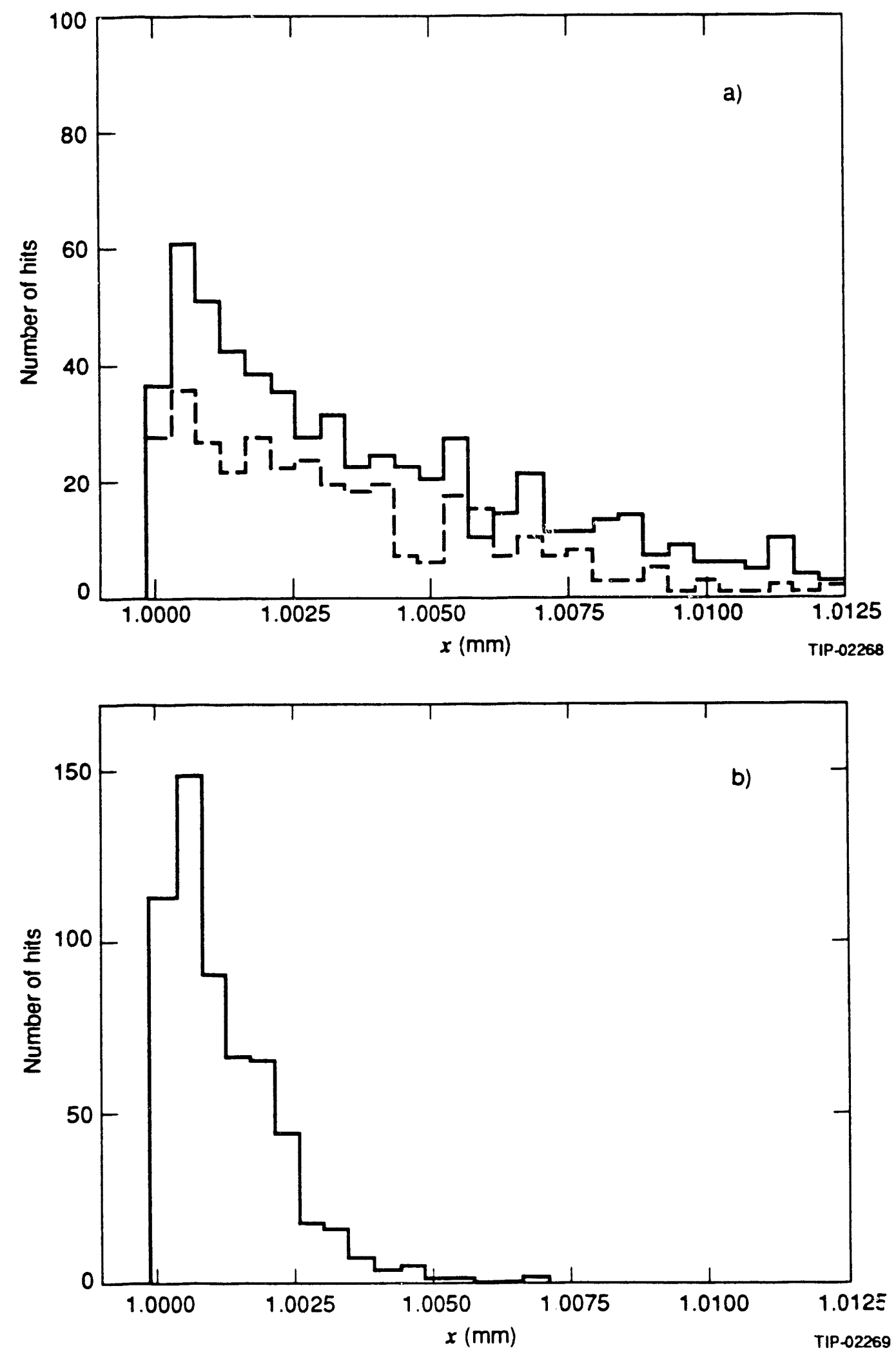

Figure 4. Distribution of Hits on Crystal. (a) $\nu_{x}=122.784$. dashes: $\beta_{x}=346 \mathrm{~m}$, solid: $\beta_{x}=1385 \mathrm{~m}$ (b) $\nu_{x}=122.501,3_{x}=1385 \mathrm{~m}$. 


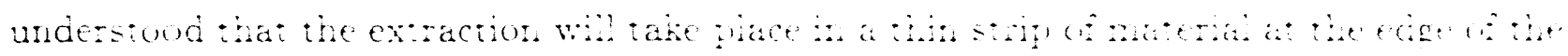

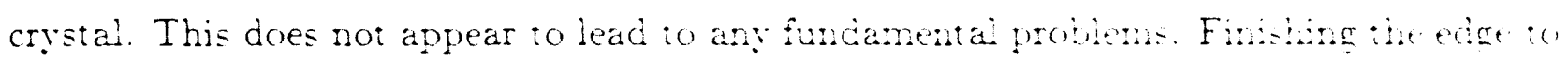
optical accuracy with disruptions of the lattice on the scale of Angstroms is consistent with

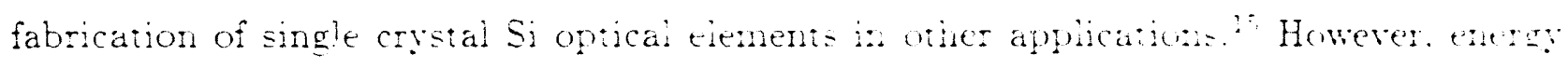
deposition and radiation damage in such a relatively small volune of crystal may impact the system design.

\subsection{SLMMARY}

The physics potential of a fixed target capability for Beauty physics at the SSC hat stimulated the interest in the extraction of a low intensity beam from the circulating pro ton bunches. The extracted beam could serve other fixed target experiments besides SFI as well as provide $20 \mathrm{TeV}^{*}$ test beams. While the initial concept is several years old. ${ }^{16}$ substantial progress has been made subsequent to the Snowmass Summer Study.1: last year. Furthermore. interest in pursuing a similar idea at the proposed Large Hadron Collider ( $L H C$ ) at CER. ${ }^{-18}$ has grown as a result. We have described in this paper some of our recent results which contribute substantially to the demonstration of the feasibility of the concept. However. there are considerations in addition to the extraction rate and preservation of the longitudinal emittance which are important to a working extraction scheme. We have already mentioned the distribution of hits on the crystal. It is also desirable from the perspective of the physics objectives that the extraction duty factor be close to unity. That is, the time dependence of the extraction rate should be ciose to the optimum meari rate of one proton/bunch/turn. It is reasonable to expect that some variation with time of the parameters such as the noise spectral density and filter bandwidths may be. useful to maintain this ratio. This research will be part of our continuing investigation of the extraction process. Furthermore. other ideas for manipulating the rf system to move protons onto the crystal are now beginning to be inveztigated. ${ }^{19.20}$ These could also be useful in developing an optimized system. A significant part of future research will be deroted to gaining a sufficient understanding of the longitudinal dynanics in the presence of these complex modulations in anticipation of the proposed experiments at the Tevatron" beginning in 199?. 


\section{ACKNOWLEDGEMENTS}

During the course of this work. we have benefited from many discussions for which we wish to thank A. Chao. R. Carrigan. R. Cogburn. B. Cox. G. Jackson. C. I. Murphy. B. Norum, S. Peggs. J. Rosenzweig and M. Syphers. Thornton Murphy provided Figure 1 for which we are grateful.

This work has been supported by the SSC Laboratory which is operated by Universities Research Association for USDOE under contract DE-AC35-89ER-40486 and is based in part upon work supported by the Texas National Research Laboratory Commission under Grant No. RGFY9134. 


\section{REFERENCES}

1. The SFT Collaboration, "An Expression of Interest in a Super Fixed Target Beauty Facility (SFI) at the Superconducting Super Collider," 25 May 1990.

2. R. A. Carrigan. Jr., "The Application of Channeling in Bent Crystals to Charged Particle Beams," in Relativistic Channeling. edited by Richard A. Carrigan and James A. Ellison, (Plenum, New York, 1987), p. 339.

3. E. N. Tsyganov. "Some Aspects of the Mechanism of a Charged Particle Penetration Through a Monocrystal," Fermilab TM-682, August 1976.

4. S. P. Møller, E. Uggerhøj, H. W. Atherton, M. Clément, N. Doble, K. Elsener, L. Gatignon, P. Grafström, M. Hage-Ali, and P. Siffert, Phys. Lett. B 256, 91 (1990).

5. B. S. Newberger and H.-J. Shih, in Nonlinear Dynamics and Particle Acceleration, Conference Proceedings No. 230. edited by Yoshi H. Ichikawa and Toshiki Tajima (AIP, New York, 1991), p. 251; "Low Intensity Beam Extraction at the SSC," SSC Laboratory Report SSCL-344, January 1991.

6. J. A. Ellison, Nucl. Phys. B 206, 205 (1982).

7. J. S. Forster, H. Hatton, R. J. Toone, G. Este, S. I. Baker, R. A. Carrigan, Jr., W. M. Gibson. R. L. Wijayawardana. J. A. Ellison, L. Emman-Wori and B. O. Kolbesen. Nucl. Phýs. B 318. 301 (1989).

8. J. L. Andersen. "Notes on Channeling," University of Aarhus, 1986 (unpublished).

9. H.-J. Shih and A. M. Taratin. "Bent Crystal Extraction of the SSC Beam with RF Noise Induceu Diffusion." SSCL Report No. SSCL-389. March 1991.

10. G. Dóme. "Diffusion due to RF Noise," CERN Advanced Accelerator School. Advanced Accelerator Physics. CERN Report No. 87-03, 1987 pp. 370-401.

11. S. Frinsky and J. M. Wang. Part. Accel. 12, 107 (1982).

12. R. Cogburn. J. A. Ellison, B. S. Newberger and H.-J. Shih. in preparation.

13. J. A. Ellison. B. S. Newberger and H-J. Shih. "Iffect of RF Phase Noise on the SSC Beam." Proc. 14th Biennial Particle Accelerator Conference. San Francisco, CA. May 6-10. 1991. to be published: also SSC Laboratory Report, SSCL-432, May 1991.

14. H.-J. Shih. J. A. Ellison. B. S. Newberger, "RF noise induced diffusion in the SSC beam." in preparation.

15. L. Berman. Personal communication. May: 1991.

16. B. Cox. J. Lach. M. Maslor. A. Mokhov, C. T. Murphy. R. J. Stefanski, in Proceedings of the Summet Study on High Energy Physics in the 1990 's. edited by Sharon Jensen World Scientific. Singapore. 19591. p. 536. 
17. H. Brown, J. Cumalat, R. A. Carrigan. Jr.. C. T. Murphy. S. Peggs. A. Garren, J. A. Ellison. J. Trischuk. D. Kaplan. B. Newberger. H.-J. Shih. T. Toohig. S. Conetti. B. Cox, B. Norum. "Report of the Super Fixed Target Beauty Facility. Working Group On Progress Towards the SFT at the SSC." Proc. 1990 Summer Study on the Physics of the SSC (Snowmass), to be published.

18. The LHB Working Group. An $R \& D$ Program for Extraction of the $8 \mathrm{TeV} / \mathrm{c} \mathrm{LHC} \mathrm{Beam}$ using Crystal Channeling. May 22. 1991.

19. S. Peggs. "Proton Mining-Dual Frequency Amplitude Modulation." Fermilab AP Note 91-001. Maỵ 9. 1991.

20. B. Norum. personal communication. March 1991.

21. R. A. Carrigan, Jr.. et al.. "Proposal for a Test of Low Intensity Extraction from the Tevatron Ľsing Channeling in a Bent Crystal," Fermilab P-853, May 22, 1991. 

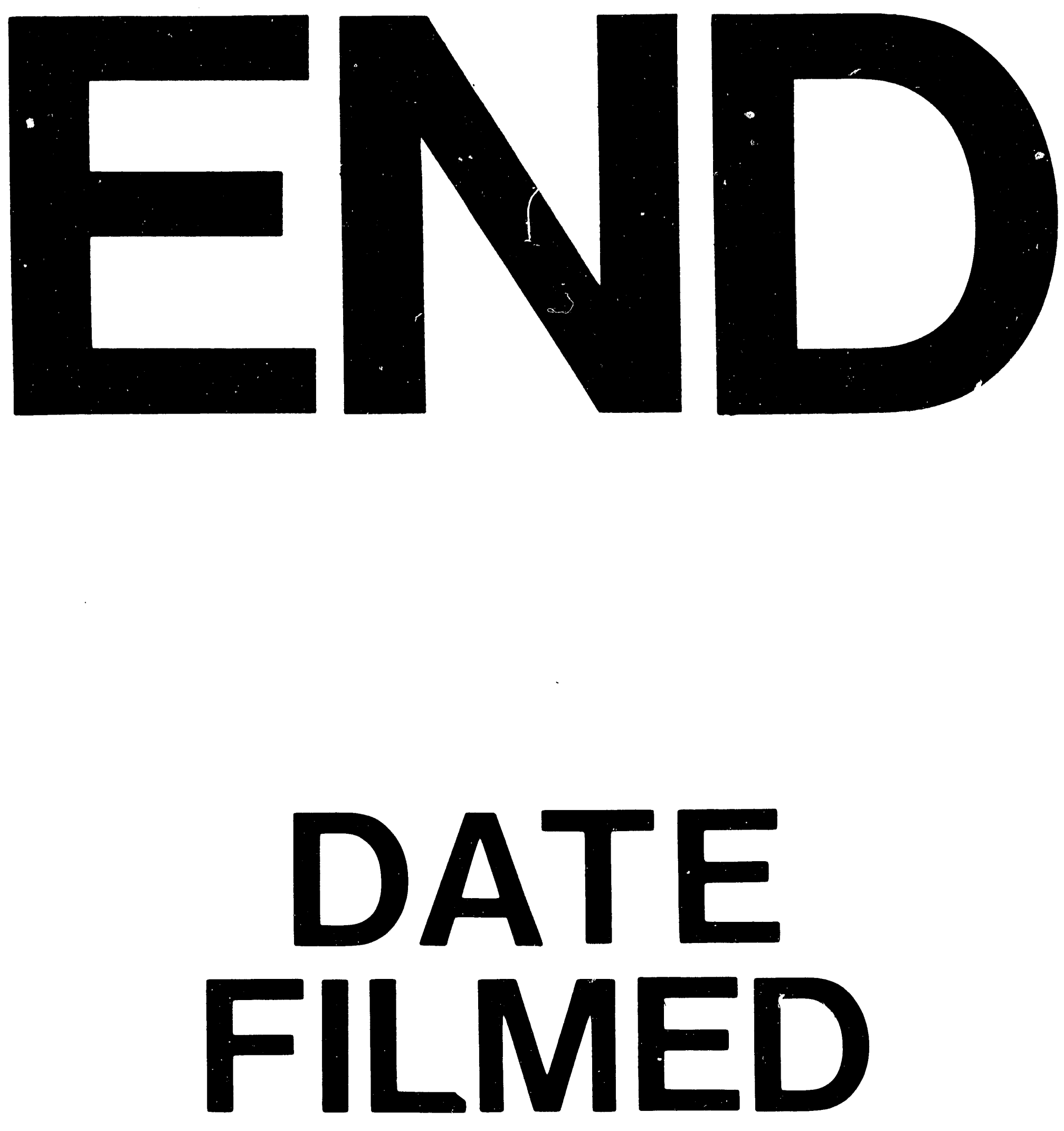

1

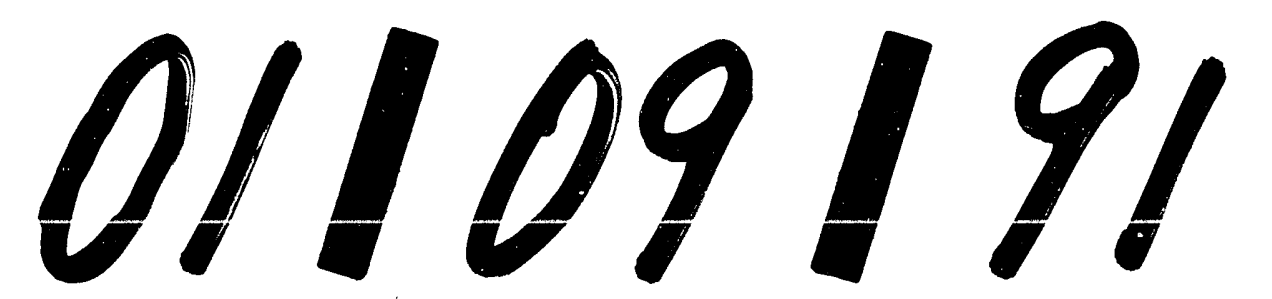


